\title{
eVIN: Role of Digitization in Improving the Efficiency of Vaccine Logistics System across India
}

\section{Shivesh Devgan ${ }^{1}$, Snehil K Singh ${ }^{2}$, Lokesh Sharma ${ }^{3}$, Setu Sinha ${ }^{4}$}

${ }^{1}$ Associate Professor, Department of Community Medicine, Sri Guru Ram Das Institute of Medical Sciences \& Research, Amritsar, Punjab, India.

${ }^{2}$ Team lead, National Cold chain and Vaccine management resource centre, NIHFW, New Delhi, India.

${ }^{3}$ MIS \& EVM Co-ordinator, National Cold chain and Vaccine management resource centre, NIHFW, New Delhi, India.

${ }^{4}$ Assistant Professor, Department of Community Medicine, Indira Gandhi Institute of Medical Sciences, Patna, Bihar, India.

Correspondence : Dr Setu Sinha, Email : drsinhasetu@gmail.com

\section{Abstract:}

Electronic Vaccine Intelligence Network (eVIN) is an indigenously developed technology which strives to digitise vaccine stocks and monitor the temperature of the cold chain through a smartphone based application. The platform supports the central government's Universal Immunization Programme by providing real-time information on vaccine stocks, flows and storage temperatures across all cold chain points of the country. This vaccine logistics system has been introduced by the Ministry of Health and Family Welfare and implemented by the United Nations Development programmers. In terms of functionality, eVIN aims to provide an integrated solution to address constraints of infrastructure, monitoring and human resource, inadequate vaccine stocks and related challenges.

Keywords : Digitization, Real Time Temperature Monitoring, Vaccine Logistics

\section{Introduction:}

In recent years, vaccines have emerged as a very significant mode of preventive intervention, providing specific protection against many communicable diseases. Various governments in the world have adopted the Universal Immunization Program (UIP) of the World Health Organization (WHO) to ensure coverage of the most vulnerable groups, comprising pregnant women and infants/children by many of these vaccines. In a nation of 1.3 billion people, it is indeed a herculean task to target 56 million pregnant women and children for immunization each year - making it the largest and most ambitious immunization program globally. ${ }^{[1]}$

\begin{tabular}{|c|l|}
\hline Quick Response Code & Access this article online \\
\hline & Website : \\
& Www.healthlinejournal.org \\
\cline { 2 - 2 } & DOI : \\
& 10.51957/Healthline_245_2021 \\
\hline
\end{tabular}

The success of the UIP resides in its ability to deliver safe and potent vaccines to its end beneficiaries. To do so, it relies on the vast network of cold chain points spread across the country. In 2015, India launched Electronic Vaccine Intelligence Network (eVIN), a smart, easy-to-use technology aimed at digitizing vaccine stocks in the country. Immunization supply chain - cold chain consists of a series of storage and transport links all of which are designed to keep the vaccine at the recommended temperature from the point of manufacture till it reaches the targeted beneficiary. ${ }^{[2]}$

Recognizing the need for a smart vaccine logistics system, the Ministry of Health \& Family Welfare (MoHFW), Government of India turned towards

\section{How to cite this article :}

Devgan S, Singh S, Sharma L, Sinha S. eVIN: Role of Digitization in improving the efficiency of vaccine logistics system across India. Healthline. 2021; 12(3):07-13. 
technology that could ensure vaccines are available at accurate location and time and in the required quantity. Implemented in collaboration with United Nations Development Programme (UNDP), eVIN is a mobile and cloud-based application that allows cold chain handlers to update information on vaccine stocks after every immunization session. ${ }^{[3]}$ These updates are stored on a cloud-based server that facilitates health officials to monitor vaccine stocks and flows. It assists the concerned personnel in decision making, pertaining to course correction, wastage reduction as well as the empowerment of health workers. The aim of this Global Alliance for Vaccines and Immunization (GAVI) supported health systems strengthening project is not just to streamline but also to regularize the network of vaccine flow. This is achieved by ensuring efficient management of the immunization supply chain by the use of authentic real time data. ${ }^{[4]}$

The goal is to ensure equity in easy and timely availability of vaccines to all children. eVIN has been digitalizing vaccine stocks at all 27,000 vaccine storage centres across all districts of 29 states and 7 union territories of India; facilitating real-time monitoring of storage temperatures by installation of nearly 50,000 temperature loggers, building capacity of nearly 37,000 government personnel for vaccine and cold chain logistic management on eVIN; deploying vaccine and cold chain managers in every district for constant supportive supervision. ${ }^{[3]}$

\section{Prominent roles of eVIN}

The prominent functions of eVIN are temperature monitoring, stock management, vaccine distribution, vaccine management practices as well as catering to beneficiaries.

\section{Temperature monitoring}

One of the prominent functions of eVIN is the real time temperature monitoring at various cold chain points. A device connected to the Ice Lined Refrigerator (ILR)/deep freezer, monitors the temperature range in which the vaccines are to be stored. Furthermore, a cloud-based data is generated which can be traced by higher centers to monitor the irregularities in temperature at all cold chain points. Thus, it results in reliable collection of temperature and power data over mobile internet/SMS transmission. An inbuilt alarm system is equipped to send SMS notification to the cold chain handlers, alerting them of temperature excursions above or below the permissible thresholds. It provides a quick overview of cold chain network to understand regional performances with drill down maps to corresponding asset detailing. It further acts as a means to double check the manual temperature recording which is taken only twice a day. Also, an average temperature reading can be visualized through the given course of time, aiding in maintaining data accuracy. Apart from all these benefits, real time notifications are received on temperature breaches, power outages and device failures. Immediate alarm system displayed on cloudbased server prompts corrective actions to be taken at the earliest from lower level (Cold Chain handlers) to the higher level (Medical Officer / District Immunization Officer). Through eVIN, temperature monitoring which was earlier on-off activity practiced only with two readings per day has been converted to a continuous task (real time monitoring) which can be carried out efficiently at each and every level.

A mechanism for cross-checking eVIN and noneVIN records has been devised to promptly identify the fault in either of the readings. Stem thermometer is being used alongside in the facility for physical inspection and standard protocol, for reading the thermometer sometimes makes it susceptible to false reading. The robust data obtained from eVIN itself stands as a proof of various temperature fluctuations undergone by the certain batch of vaccines. On this basis, a cloud-based data can be generated giving a projected view of the temperature variations subjugated via eVIN. A predictable pattern of cold chain system functioning (cleaning, defrosting, 
equipment failure) can be viewed and actions taken on timely basis. Owing to real time recording of temperature, it helps to monitor the guideline protocol compliance being processed by cold chain handlers and in case of mishaps, it can be rectified in a timely manner. Installation of temperature loggers on site has proved even more beneficial in case of remotely located cold chain points. Even on the holidays, the alarm system makes up for assessment which is done through physical monitoring. An hourly notification of separate hot and cold temperature alarms has helped in regular monitoring of Cold chain equipment and prevention of vaccine wastage. A proper functioning of real time monitoring further has strengthened the implementation of "Open vial policy" which has ensured both the quality and potency of vaccines in the Cold Chain Equipment (CCE) by regular monitoring of storage temperatures, equipment health, status of equipment defrosting, reverse cold chain and alternate vaccine delivery system.

\section{Stock Management}

The idea behind vaccine stock management in immunization is basically to estimate the quantum of vaccines required to timely immunize the target population. Good stock management practices require accurate consideration of the type of vaccine, the presentation (vial size), the quantity and timely delivery of the vaccine. Considerable improvement can be done on the grounds of maintaining minimum \& maximum stocks, as well as addressing stock out or excess stock events. It is important to ensure that the cold chain is not overburdened as well as not under supplied. The minimum stock level of vaccines is the level below which the stock should never drop without having placed an order, which is known as "reorder level".

Overstocking and stock-outs of vaccines may occur because of the lack of real-time vaccine stock visibility, weak inventory and stock-flow record keeping practices; and the absence of distribution planning by qualified personnel. Moreover, the temperature monitoring of CCE is largely dependent on the availability of a dedicated human resource at the Cold Chain Point (CCP). This type of system maintenance poses serious challenges to the quality of recording, reporting and monitoring temperature of CCEs. In addition, there are several issues pertaining to poor record keeping, use of nonstandardized stock registers and distribution practices.

Number of facilities having stock-out of any vaccine showed a remarkable reduction by $30 \%$ in post-eVIN period $(\mathrm{p}<0.001) .40 \%$ less instances of stock-out per facility was observed after eVIN implementation $(p<0.001)$. Average duration of stock-out reduced from 13 to 8 days per facility in an observation period of six months in the post eVIN period $(\mathrm{p}<0.001)$. Maximum reduction in number of days of stock-out/facility was observed for Measles (72\%), followed by Hepatitis B (65\%) and Pentavalent (62\%). ${ }^{[5]}$

eVIN aids in optimizing stock availability across all warehouses and last-mile stores with the intention of incurring minimal inventory holding costs. One can easily monitor the availability and storage facilities using real time data. In this mobile app, necessary columns which are critical indicators are given in a tabulated manner ensuring their entries to be filled while filling the indent form. Indent form is used to generate indent of vaccines required to conduct immunization sessions or to cover up the demand in case of stockout/minimum stock of vaccines. On the implementation of eVIN, critical indicators (amount demanded, closing balance, total amount received, date of indent, etc.) are not missed thereby increasing the completeness of indent form. A detailed mention of inventory item is captured through eVIN such as batch ID, details of the higher center from which the vaccine has been received and expiry date. Issue voucher is generated enlisting the type of antigen required, its quantity, opening and closing stock, net amount in rupees, updated details mentioning date and person who has 
updated the record. Over 2 million transactions are being made on eVIN every month capturing essential information such as vaccine issues, receipts discards and transfers. More than 14,000 data loggers have been installed for real time remote temperature monitoring of cold chain equipment. ${ }^{[6]}$ Thus, a record of inventory issue vouchers, receipts, stock counts and returns are kept safe under this mobile application and receive notifications from time to time on stock changes as and when they happen.

It is a very good method to optimize inventory by cross verifying with the physical count where any discrepancy if found can be easily sorted out at the earliest. In this manner, rebalance stock ensures good service level thereby preventing wastage. The detail of entire inventory is displayed in the form of map where region wise monitoring of stock levels can be done and necessary action can be taken if in case CCP to CCP sharing of vaccine is required. Another advantage which eVIN presents us with is the ability to capture new data in case newer vaccines are introduced (for instance, MR campaign). In these situations, a new column can be created through eVIN and the entire campaign stock record can be managed paralleled with the ongoing immunization sessions.

Another critical finding has been the reduction in avoidable wastage of vaccine doses (unopened vials). The secondary data analysis of UNDP records suggests that the facilities reporting wastage of any vaccine have reduced from $30 \%$ in pre-eVIN period to $22.6 \%$ in the post-eVIN period, indicating a $25 \%$ reduction in wastage of any vaccine due to reasons such as non-usable vaccine vial monitor (VVM), freezing, expiry and broken vials (data source: UNDP reported eVIN and VCCM records). The highest reduction in avoidable wastage was observed for DPT (reduced by 98.7\%) and the lowest was observed for OPV (reduced by 21.9\%) after the implementation of eVIN. ${ }^{[5]}$

\section{Vaccine distribution}

Another significant impact of eVIN can be seen in its marked reduction in mean replenishment time across all cold chain facilities. Replenishment time is the difference between date of supply received and date of indent raised to the higher center. As a metric of inventory management, order fill rate is quite essential to be noted, which is, the proportion of quantity received to that of quantity indented. This is basically a measure of percentage of demands that were met at the time the order was placed. Although order fill date is not considered to be a true reflection of the improvement in efficiency in vaccine distribution because sometimes it so happens that various instances are recorded which reflect excess distribution by the higher centers in comparison to the actual demand placed by the lower centers, although in reality it is the compensation of previous backlog. In this manner, the true picture of missed opportunities/missed sessions is not depicted. So, a better utilization with the help of eVIN will guide us in recognizing the actual distribution coverage of the given area.

The distribution system is a mix of push and pull across all levels of immunization supply chain. The effectiveness of a distribution system lies in lesser replenishment time, complete order fill rate and minimal expiry of vaccines at stores. In pre-eVIN period, it took seven days in getting supplies from a higher-level store, which has now reduced to three days, even though, order fill rate remained almost unchanged (from $97 \%$ in pre-eVIN to $99 \%$ in posteVIN). Furthermore, mean number of days (of vaccine expiry) has reduced from 428 in pre-eVIN to 384 in the post-eVIN, at CCP level signifying that the 'First Expiry First Out' (FEFO) is being practiced. ${ }^{[5]}$

In India, usually two sessions are conducted in a duration of seven days, and the number of sessions missed are in proportion of the number of Auxiliary Nurse Midwives (ANM) available at a specific health facility. Based upon this information, the number of 
sessions missed due to stock-outs used to be calculated for each of the antigens separately. If the number of days of stock-out of an antigen lasted for a minimum of three days, it was assumed that one session was missed. Two sessions were counted missed if the stock-out lasted for a minimum of another four days. Similarly, next session is considered missed if the stock-out lasted for another three complete days and next in another four days and likewise. The sum of missed sessions at a facility was in proportion of the total sessions conducted in the same duration at that facility. Based on the sum of days of stock-outs of vaccines, subsequent missed opportunities were calculated.

The number of beneficiaries missed due to stockout of an antigen was calculated using Health Management Information System (HMIS) data of the same duration. The average number of beneficiaries vaccinated in a session was generalized from HMIS data for each of the district and was assumed that the same numbers of beneficiaries were vaccinated at the facility as well. The sessions missed were multiplied with the number of beneficiaries vaccinated in a session at a facility.

With the introduction of eVIN, there have been evidences which suggest marked savings in vaccine utilization which can be largely attributed to open vial policy. Proper tracking of the vaccine utilization and daily record upkeep by eVIN has led to significant reduction in utilization of doses that aids in various vaccine supply strengthening measures. The vaccine utilization data of Immunization Division, MoHFW reflects that the utilization has reduced from 3,053 lakh doses in pre-eVIN period to 2,149 lakh doses in post-eVIN period across 12 eVIN states, resulting into savings of approximately 900 lakh ( 90 million) doses of vaccines. ${ }^{[3]}$ These savings can be attributed to the roll-out of eVIN across 12 states, and other initiatives such as the introduction of open vial policy, effective vaccine management assessments, and continuous follow-up with an improvement plan in place. ${ }^{[5]}$ The roll-out of eVIN has effectively improved the visibility of stock till last cold chain point and has also led to better vaccine management practices such as reduction in vaccine wastage and stock-out events. As we can find out that there occurs CCP to CCP sharing of vaccines at operational level, this effort can be made more streamlined through eVIN which can reduce the horizontal sharing and if the sharing occurs, it more or less compensates the actual requirement of the recipient CCP in terms of replenishment time or order fill date.

\section{Stock Management practices}

There has been a significant achievement in terms of stock management practices when it comes to comparison of pre and post eVIN periods. Various parameters in which stock management is required, eVIN has proved quite handy in streamlining all those parameters without any extra input from its end.

As, eVIN registers all the indicators in the different fields by physical count, the completeness of the record increases and missed entries are reduced. In addition, the indent generated from eVIN covers the entire demand/supply of vaccines required to conduct immunization sessions. As a result, a systematic record is maintained containing all the information about inventory details like opening stock, amount received, source of stock, batch number, expiry, closing balance, VVM status, etc. Tallying vaccine-wise stock is further enhanced by carrying out comparison between stock register and eVIN record and another between eVIN record and physical count.

A standard log book register is provided to the cold chain handlers in which session wise vaccine dispensing record is updated after the completion of each session. Thus one can access data digitally as well as manually, in a periodical manner and troubleshoot whenever needed. It also ensures timely upkeep of cold chain equipment by mentioning dates for servicing or replacement.

For stock management, use of standardized stock registers by CCHs has increased from $56 \%$ in pre- 
eVIN to $97 \%$ in post-eVIN period. The updating of vaccine stock register (either daily or weekly) has improved from $54 \%$ in pre-eVIN to $81 \%$ in the posteVIN period, reflecting an overall improvement in vaccine management practices. ${ }^{[6]}$

An essential component of successful vaccine logistics is based upon potent and safe to use cold chain system. The completeness of record keeping was checked against applicable fields of the register. The vaccine stock registers, having more than $90 \%$ completeness, has increased from 29\% facilities in pre-eVIN period as compared to $75 \%$ facilities in the post-eVIN period. The temperature log books, with almost all the fields completed has increased from $28.5 \%$ facilities to $69.8 \%$ facilities. Similarly, indent registers with almost all fields completed, has increased from $26 \%$ facilities to $69 \%$ facilities in the observation period. During accuracy check across 12 states, 94\% accuracy was observed in the stock registers and eVIN entries. Similarly, 92\% accuracy was observed in the physical counting of doses and eVIN entries. ${ }^{[7]}$

\section{Avenues}

Apart from the mobile app, eVIN can help us in realistic estimation of target consumption noted at each CCP, giving us a forecasting view of the number of beneficiaries per area. There are various avenues in which eVIN can indirectly be a guiding light in terms of preparing the micro plan, assess the number of sessions missed, also help in decreasing the number of missed opportunities. Furthermore, monthly/annually pattern of demand can be seen and the estimate to that of actual requirement of vaccines can be assessed.

Though eVIN is conducting Temperature Mapping Study at Primary Vaccine stores before installing their temperature monitoring devices. However, Temperature monitoring study and Temperature mapping study can also be conducted at all PVSs. Technical agencies of GOI may support the states in conducting these studies. Also, a detailed SOPs as per WHO recommendation may be prepared.
Engagement of partner agencies for hands on training can also be done. ${ }^{[8]}$

\section{Challenges}

Countrywide implementation of eVIN is an arduous task. As of now, cold chain is catering to a total of 11 vaccines, namely Bacillus CalmetteGuerin (BCG), Hepatitis B, Oral Polio vaccine (OPV), Pentavalent vaccine, Pneumococcal Conjugate Vaccine (PCV), Rotavirus Vaccine (RVV), Inactivated Polio Vaccine(IPV), Measles \& Rubella (MR), Japanese Encephalitis (JE), DPT and Td ${ }^{[9]}$ For keeping the vaccines potent at recommended temperature, India's UIP program has various electrical and nonelectrical cold chain equipment. In electrical CCE, India has Walk in Cooler, Walk in Freezer, Ice Lined Refrigerator, Deep freezer etc. In non-electrical CCE India has Cold box and vaccine carrier. ${ }^{[10]}$ Apart from requiring huge amounts of equipment, human resource training remains the biggest challenge as this requires huge input in terms of recruitment and training of the vaccine cold chain handler. Differences in uptake of vaccine are geographical, regional, ruralurban, poor-rich and gender-related. As for example, on average, girls receive fewer vaccinations than boys and higher birth order infants have lower vaccination coverage. Some of the newer challenges in achieving full immunization coverage include limited capacities of staff, particularly in poor-performing states and at the field level and gaps in key areas such as predicting demand, logistics and cold chain management, which result in high wastage rates. India also lacks a robust system to track vaccinepreventable diseases. ${ }^{[11]}$ Any roll out of technology, without training the frontline health workers, can never lead to building up their confidence in the same. Hence, training, sometimes with personal attention becomes an important pre-requisite for the successful roll out of the technology. A continuous electricity supply for uninterrupted and efficient real time monitoring is much needed in order to reap maximum benefits of eVIN app. The program governance has to time and again assess its 
implementation as well as operative costs in order to ensure low cost benefit ratios.

\section{Conclusion:}

Real time monitoring of the vaccine temperature and stock inventory is a great managerial tool for vaccine program managers. eVIN gives an overview of the entire vaccine distribution pattern and assists in forecasting future needs, stocking and replenishment patterns. Furthermore, any delay due to a specific cause may also be highlighted in eVIN, for which a corrective measures can be sought. This way a contingency plan at the grassroot level can be planned while giving valuable feedback to the administrators.

Given the advantages of eVIN, proper implementation of eVIN can streamline vaccine logistics system. Also, worth exploring are avenues regarding batch recognition, transit tracking and inbuilt order fill rate notifications etc. This technology needs a thorough utilization by our health professionals so that this technology blends into our inbuilt system of robust vaccine management and its functionality can be enhanced that it becomes a daily affair to reach out to this mobile app on the go, whenever required for. This robust system has been used with the requisite customization during the COVID pandemic for ensuring continuation of the essential immunization services and protecting children and pregnant mothers against vaccine preventable diseases.

\section{Declaration:}

\section{Funding: Nil}

\section{Conflict of Interest: Nil}

\section{References:}

1. Immunization Division, Ministry of Health \& Family Welfare, Government of India (MoHFW-GoI). Comprehensive Multi-Year Plan 2018-22. New Delhi: MoHFW-GoI; 2018.p 2. [last assessed on 2021 June 11]. Available from: https://nhm.gov.in/ New_Updates_2018/NHM_Components/Immunization/Guildeli nes_for_immunization/cMYP_2018-22_final_pdf

2. National Cold Chain Vaccine Management Resource CenterNational Institute of Health \& Family Welfare (NCCVMRC-
NIHFW) \& UNICEF. National Effective Vaccine Management Assessment. New Delhi: NCCVMRC-NIHFW \& UNICEF; 2018. p 5. [last assessed on 2021 June 12]. Available from: https://nhm.gov.in/New_Updates_2018/NHM_Components/Im munization/Guildelines_for_immunization/National_EVM_Assess ment_Report_2018.pdf

3. United Nations Development Programme, India (UNDP-India). Improving the efficiency of vaccinations systems in multiple states [Internet]. New Delhi: UNDP-India. [last assessed on 2021 June 13] Available from: https://www.in.undp.org/content /india/en/home/projects/gavi1.html

4. Muraskin W. The Global Alliance for Vaccines and Immunization: is it a new model for effective public-private cooperation in international public health? Am J Public Health. 2004 Nov;94(11):1922-5. doi: 10.2105/ajph.94.11.1922. PMID: 15514228; PMCID: PMC1448560.

5. Ministry of Health \& Family Welfare, Government of India (MoHFW-GoI). Techno-Economic Assessment of Electronic Vaccine Intelligence Network. New Delhi: MoHFW-GoI; 2018.[last assessed on 2021 June 15]. Available from: https://nhm.gov.in/New_Updates_2018/NHM_Components/Im munization/Guildelines_for_immunization/eVIN_Assessment_Re port.pdf

6. Immunization Division, Ministry of Health \& Family Welfare, Government of India (MoHFW-GoI). Comprehensive Multi-Year Plan 2018-22. New Delhi: MoHFW-GoI; 2018. p 18.[last assessed on 2021 June 11].Available from: https://nhm.gov.in /New_Updates_2018/NHM_Components/ Immunization/ Guildelines_for_immunization/cMYP_2018-22_final_pdf

7. Gurnani V, Singh P, Haldar P, Aggarwal MK, Agrahari K, Kashyap S, et al. (2020) Programmatic assessment of electronic Vaccine Intelligence Network (eVIN). PLoS ONE 15(11): e0241369.https: //doi.org/10.1371/journal.pone.0241369

8. National Cold Chain Vaccine Management Resource Center- National Institute of Health \& Family Welfare (NCCVMRC-NIHFW) \& UNICEF. National Effective Vaccine Management Assessment. New Delhi: NCCVMRC-NIHFW \& UNICEF; 2018. p 30. [last assessed on 2021 June 12]. Available from: https://nhm.gov.in/ New_Updates_2018/ NHM_Components/Immunization/Guildelines_f or_immunization/ National_EVM_Assessment_Report_2018.pdf

9. Universal Immunisation Programme: National Health Portal of India [Internet]. [last assessed on 2021 Jul 15]. Available from: https://www.nhp.gov.in/universal-immunisation-programme_pg

10. Ministry of Health \& Family Welfare, Government of India-UNICEF. Immunization Handbook for Vaccines \& Cold chain handlers. $2^{\text {nd }} e d$. New Delhi: Dept. Health \& Family Welfare, Govt. of India, 2016. p 23-36. [last assessed on 2021 Jul 16].Available from: https://www.technet21. org/media/com_resources/trl/2244/ multi_upload/Unicef_Cold\%20Chain\%20Handlers\% 202016.pdf

11. Immunization: UNICEF India. [Internet]. [last assessed on $2021 \mathrm{Jul}$ 15]. Available from: https://www.unicef.org/india/what-wedo/immunization 